\title{
CATEGORÍAS DE ANÁLISIS PARA LA INVESTIGACIÓN DE LA REPRESENTACIÓN INFORMATIVA DE LAS MANIFESTACIONES SOCIALES
}

\author{
Dorde Cuvardic \\ Eduardo Cordero
}

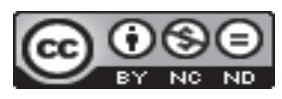

Esta obra está bajo una licencia Creative Commons Reconocimiento-No Comercial-Sin Obra Derivada 



\title{
CATEGORÍAS DE ANÁLISIS PARA LA INVESTIGACIÓN DE LA REPRESENTACIÓN INFORMATIVA DE LAS MANIFESTACIONES SOCIALES
}

\author{
CATEGORIES OF ANALYSIS FOR THE RESEARCH OF \\ INFORMATIVE REPRESENTATION OF \\ SOCIAL DEMONSTRATIONS
}

\section{Dorde Cuvardic Eduardo Cordero}

\begin{abstract}
RESUMEN
El siguiente artículo es el resultado de una investigación cualitativa de tipo exploratoria que se planteó la siguiente pregunta de investigación: ¿Qué procedimientos discursivos verbales y visuales son comunes en la representación informativa de las manifestaciones? Dar respuesta a esta interrogante permitió identificar las categorías de análisis de la representación informativa de las manifestaciones de los movimientos sociales, particularmente en La Nación. Se analizó e interpretó un grupo de noticias que tematizaron las manifestaciones realizadas en contra de la aprobación del Tratado de Libre Comercio con Estados Unidos (publicadas entre el 2003 y el 2007).

Palabras clave: análisis del discurso, Tratado de Libre Comercio Costa Rica-Estados Unidos, categorías de análisis, representación informativa, movimiento social.
\end{abstract}

\begin{abstract}
The following article is the result of an exploratory qualitative research that posed the following question: What verbal and visual discursive procedures are common in the informative representation of social demonstrations? This question allowed us to identify the categories of analysis of the informative representation of social demonstrations, particularly in La Nación. We analyzed and interpreted a group of news published between 2003 and 2007 that dealt with the demonstrations against the approval of the Free Trade Agreement with the United States [or The Dominican Republic-Central America Free Trade Agreement (CAFTA-DR)].

Key words: discourse analysis, Free Trade Agreement between the United States and Costa Rica, categories of analysis, informative representation, social movement.
\end{abstract}

Dr. Dorde Cuvardic García. Universidad de Costa Rica. Profesor de la Escuela de Filología, Lingüística y Literatura. Costa Rica.

Correo electrónico: dcuvardic@yahoo.es

Dr. Eduardo Cordero Cantillo. Docente y Productor Audiovisual de la Universidad Estatal a Distancia. Costa Rica. Correo electrónico: ecordero@uned.ac.cr

Recepción: 13- 03- 2017

Aceptación: 08- 06- 2017 


\section{Introducción}

Las manifestaciones o marchas de los movimientos sociales (o "acciones colectivas") constituyen uno de los principales hechos -temporalmente puntuales- del espacio público de discusión. Son objeto de representación informativa, en lo que se conoce como relato informativo orientado hacia el acontecimiento, frente a aquellas noticias estructuradas a partir del tema, del asunto o del proceso social. En este sentido, debe diferenciarse la representación informativa de una huelga y la de una manifestación. Este último es un suceso temporalmente puntual incorporado en procesos sociales que se desarrollan a largo plazo, como son los conflictos sociopolíticos canalizados a través del poder legislativo. Algunos de estos últimos son iniciados por las llamadas acciones colectivas o movimientos sociales.

Una vez que logran incorporar su temario en el espacio público de discusión, los movimientos sociales políticamente hegemónicos, frente a aquellos considerados como marginales o emergentes, previsiblemente obtendrán mayor respaldo ideológico de las empresas periodísticas. Como señala Tuchman (1983, p. 147) en su clásico estudio, los movimientos sociales "que llevan las riendas reconocidas del poder legitimado tienen claramente más acceso a los medios que aquellos que no las llevan". Si pensamos en términos de paradigmas de investigación de los procesos de producción de la información, la representación de las manifestaciones se investiga desde el paradigma del newsmaking (contrucción de la noticia), frente al paradigma del gatekeeping. Los sucesos no son filtrados por los guardabarreras (gatekeepers), sino que son construidos discursivamente, y esto último ocurre con las manifestaciones.

El estudio de la representación informativa de las manifestaciones se justifica, en primer lugar, por la relevancia informativa que las empresas periodísticas otorgan a este tipo de acontecimientos. Además, la imagen que proyecten los mass media de los movimientos sociales orientará, parcialmente, el nivel de respaldo ciudadano hacia las reivindicaciones presentadas. Los medios informativos otorgan diversos grados de noticiabilidad a las acciones colectivas y, en el marco de los ciclos de protesta, a las manifestaciones. Entre otros, los valores-noticia que atribuyen a las marchas son (a) el número de participantes en el acontecimiento y (b) cercanía geográfica o sociocultural, en relación con la cotidianeidad de los lectores. Bajo estos presupuestos, el acontecer político nacional, frente al extranjero, cuenta con un mayor grado de noticialibidad. Además, los medios de comunicación les otorgan una alta cuota de relevancia a las marchas, es decir, aparecen en portada y son objeto de evaluación en los géneros de opinión (editoriales, artículos, columnas, fotografías) y quedan, por lo general, representadas en la primera página de la sección de "Nacionales" o de "Política".

Como pregunta de investigación principal, planteamos: ¿Qué procedimientos discursivos verbales y visuales son prototípicos en la representación informativa de las manifestaciones? Nuestro objetivo consiste en identificar y explicar las categorías empleadas en la construcción de la representación informativa de las manifestaciones de los movimientos sociales. Nuestra propuesta tiene, en este sentido, carácter inductivo. En particular, analizaremos e interpretaremos las noticias sobre las manifestaciones realizadas en contra de la aprobación del Tratado de Libre Comercio con Estados Unidos publicadas entre el 2003 y el 2007 en el periódico La Nación. Nuestros objetivos específicos consisten, en primer lugar, en analizar los procedimientos discursivos verbales utilizados en la representación informativa de las manifestaciones en contra del TLC en La Nación durante el ciclo de movilizaciones entre el 2003 y el 2007; en segundo 
lugar, los procedimientos visuales empleados, y, en tercer lugar, la interacción entre estos procedimientos discursivos visuales (fotografías e infografías) y los verbales. Utilizaremos, como principal estudio de caso, la noticia "Trabajadores marcharon contra el TLC", La Nación, publicada el 1 de junio de 2004. Esta noticia será analizada e interpretada a partir de la edición impresa; hemos accedido a las demás noticias, en las que se representan otras manifestaciones o marchas, desde la edición digital.

\section{Estado de la cuestión}

Las primeras investigaciones emprendidas en el ámbito de las relaciones entre los movimientos sociales y los mass media informativos proceden del ámbito anglosajón. En particular, se dedicaron a estudiar las relaciones de codependencia o conflicto entre las empresas periodísticas y las acciones colectivas a lo largo de los ciclos movilizadores (véase, al respecto, para obtener una visión panorámica, Cuvardic García, 2003). Una de las primeras procede de Gaye Tuchman (1983), desde el paradigma del newsmaking; esta investigadora comprobó que las relaciones continuadas entre los medios periodísticos y el movimiento feminista estadounidense, en los años setenta, contribuyó a otorgar una mayor institucionalización a este último, factor que incidió, al mismo tiempo, en una menor "radicalización" de sus objetivos. Se produjo una situación paradójica: el movimiento tuvo que sacrificar la defensa de objetivos "radicales" (es decir, alternativos al status quo) en favor de una mayor visibilidad mediática y de una mayor representación positiva -favorable a sus intereses- en los espacios informativos. A las mismas conclusiones llegó Todd Gitlin (1980), quien, por su parte, investigó el proceso de incorporación massmediática que tuvo el movimiento de la 'New Left' en Estados Unidos durante los años sesenta; en particular, estudió la representación informativa del Students for a Democratic Society $(S D S)$ en 1965 por parte del New York Times y de la CBS, aunque también se dedicó en el último capítulo a indagar en las relaciones que los movimientos contrarios a la energía nuclear y a las armas nucleares desarrollaron con los mass media en los años setenta.

Como vemos, la construcción discursiva informativa se ha centrado en las acciones colectivas, en los procesos o ciclos reivindicativos desarrollados en el tiempo por las organizaciones civiles, más que en la representación puntual de las manifestaciones o marchas. Además de los casos señalados hasta ahora, se ha investigado, por ejemplo, la construcción mediática de los movimientos antinucleares (Gamson y Modigliani, 1989; Entman y Rojecki, 1993), los movimientos de mujeres (Van Zoonen, 1992; Terkildsen y Schnell, 1997; Ashley y Olson, 1998) o los movimientos sindicalistas del ámbito industrial (Hartmann, 1979; Douglas, Pecora y Guback, 1985; Walsh, 1988) y del agropecuario (Marshall, 1977; Rada, 1977). Son estudios que se ocupan de indagar los temas que los medios de comunicación encuadraron (to frame) a la hora de comprender los ciclos de protesta de las acciones colectivas. Por ejemplo, Gamson y Modigliani (1989, p. 1037) estudiaron la incidencia de los marcos o encuadres (frames) temáticos a favor de la energía nuclear -(a) la energía nuclear como fuente de progreso y (b) como fuente de independencia energética- y de los marcos o encuadres en contra, sustentados por la coalición respectiva de actores -(a) la energía nuclear como un trato diabólico que conlleva consecuencias nefastas, (b) un "caballo desbocado" que es difícil controlar, (c) una energía contraria al ejercicio de la responsabilidad social, (d) una energía que conlleva costes sin ninguna eficacia y (e) la necesidad de elegir otras vías energéticas no dañinas-. ${ }^{1}$ Terkildsen y Schnell (1997) también ofrecen una propuesta 
conceptual-metodológica del encuadre temático; concluyeron que, en la prensa estadounidense, desde los años cincuenta hasta los noventa, la construcción mediática de los movimientos de mujeres y el feminismo se expresó en seis esquemas cognitivos: el feminismo, el económico (puestos laborales), el rol político de las mujeres, el anti-feminismo, los papeles tradicionales de género y las divisiones internas entre estos grupos. ${ }^{2}$ Todas estas investigaciones se centran en los framing temáticos -perspectivas para comprender un asunto social- utilizados por las acciones colectivas durante el desarrollo de los procesos conflictivos que protagonizaron en la arena pública. Debe señalarse, al respecto, que las investigaciones sobre el framing surgieron en el ámbito de la sociología de las acciones colectivas, con términos acuñados en esta última disciplina como master frames (Swart, 1995), o master frame alignment (Swart, 1995; Snow y Benford, 1988), para indagar en los determinantes de los ciclos movilizadores y, de ahí, pasaron a las investigaciones sobre las representaciones mediáticas de las acciones colectivas.

Las investigaciones mencionadas se refieren a los ciclos de protesta de los movimientos sociales. No aluden a uno de los acontecimientos más importantes de las acciones colectivas: las manifestaciones o marchas. Creemos que existe una escasez de estudios sobre la representación informativa de las manifestaciones que nuestra investigación viene parcialmente a llenar. Sobre todo, los investigadores que se han ocupado hasta ahora de las manifestaciones han estudiado el framing mediático y han llegado a determinar un encuadre negativo massmediático hacia este tipo de acontecimientos. McLeod y Detenber (1999), quienes han estudiado los efectos, en la audiencia, de la representación informativa televisada de la protesta social en Estados Unidos, se refieren al paradigma de protesta (protest paradigm) para referirse al desempeño de todos aquellos medios que deslegitiman o marginalizan, mediante un trabajo de focalización, las reivindicaciones de grupos sociales que desafían el status quo: "las noticias sobre protestas tienden a centrarse en la apariencia de los manifestantes, antes que en los asuntos reivindicados, y destacan las acciones violentas, antes que la crítica social planteada" (p. 3).

Los estudios mediáticos sobre la representación informativa de los ciclos de protesta y las marchas o manifestaciones que surgieron inicialmente en el ámbito anglosajón, también se han incorporado a la agenda investigativa de España y América Latina. En el ámbito comunicativo, Castillo-Esparcia, García-Ponce y Smolack-Lozano (2013), por medio del análisis del mensaje de los siete periódicos de mayor difusión de España y de su presencia en las redes sociales, concluyeron que la representación informativa del movimiento anti-sistema español del 15-M se centró más en las acciones, las movilizaciones y las demandas políticas desde este actor social, aunque desde una escasa profundidad analítica, mientras que la representación informativa de Ocuppy Wall Street contó con una mayor presencia de expertos (en su papel de fuentes) y de las demandas presentadas (reformas económicas). LafuentePérez y Zugasti-Azagra (2012) también analizaron el movimiento del 15-M, específicamente en los diarios El País y El Mundo, desde los protagonistas, fuentes y temas. Concluyeron que las reivindicaciones económicas del movimiento quedaron visibilizadas en segundo plano, frente a la evolución de la campaña y de las movilizaciones de este actor social. Piquer-Vidal (2013) analizó la representación del movimiento estudiantil chileno del 2011 en el diario Las últimas noticias y de su líder, Camila Vallejo, que recibió una representación sexista por parte del medio de comunicación. Empleó un análisis muy común en los estudios sobre el discurso mediático, consistente en investigar las macroproposiciones y los significados locales explícitos e implícitos de las noticias. Por último, Morales-Morante (2014) analizó 
la representación televisiva de dos huelgas de maestros, la primera realizada en España y la segunda en Perú; concluyó que, aunque en ambos casos las noticias se encuadraron desde la perspectiva gubernamental, en el primer caso se estructuró un formato más perspectivístico (se visibilizaron las diversas posturas en conflicto), mientras que, en el segundo, se procedió a una mayor espectacularización de la huelga y se focalizó la atención en las acciones violentas ocurridas. Por último, Acosta (2013) no analizó exactamente la perspectiva periodística del movimiento de izquierdas colombiano Marcha Patriótica, sino las declaraciones hegemónicas (sobre todo, las procedentes del ejército) que deslegitimaban sus objetivos. Si sintetizamos e interpretamos globalmente los resultados de estas investigaciones, llegamos a las siguientes conclusiones. En primer lugar, son análisis del mensaje periodístico (análisis de temas o macroproposiciones, actores, fuentes, focalización). No se inscriben en un análisis crítico del discurso de carácter lingüístico. Analizan la representación informativa de un movimiento social o de una huelga (en su proyección social, en sus campañas, en sus fuentes, en sus movilizaciones). No estudian estrictamente la representación informativa de una marcha o manifestación específica (inscrita, por lo general, en un ciclo de protesta de un movimiento o de una huelga). En pocas ocasiones se analiza la representación informativa desde la perspectiva del framing, propuesto por el medio o por los actores protagonistas.

Las fotografías y el formato televisivo también forman parte importante de la representación informativa de las manifestaciones. Han sido objeto de atención por parte de Cuvardic García (2004-2005) los temas de las fotografías de la representación informativa en la huelga de educadores en Costa Rica en el 2003: (a) fotografías de manifestaciones públicas de protesta, (b) de reuniones negociadoras, (c) de conferencias de prensa, (d) de reuniones de los actores reivindicativos (o de los grupos de interés movilizados), (e) de las campañas y de las actividades comunicativas ante la ciudadanía, y (f) de los afectados por las huelgas, están entre los temas fotográficos más importantes identificados. Además, entrevistó a reporteros gráficos para conocer los determinantes que se encuentran detrás de la publicación de las fotografías en los medios impresos. Por su parte, López del Ramo y Torregrosa (2011, pp. 277-290), en su investigación sobre los procedimientos denonativos y connotativos de las fotografías publicadas en La Vanguardia, El País, El Mundo, ABC y La Razón, sobre la huelga general del 29 de septiembre de 2010 en España, concluyeron que, si bien los gestos de los manifestantes fotografiados - una de las categorías de análisis empleadas- no respondieron al estereotipo del sujeto violento, fue mostrada esta actividad como una acción con un alto componente de tensión. Por último, Velásquez (2004) investigó la representación informativa televisiva colombiana de dos protestas, la primera a raíz de problemas en el servicio transmilenio, en los noticieros City Noticias y CM\& (ediciones de las 7:45 p.m. y 9:30 p.m. del 9 de marzo de 2004), y la segunda motivada por el inicio de las negociaciones para establecer un Tratado de Libre Comercio con Estados Unidos, en los noticieros Caracol y RCN (edición de las 7:00 p.m.). En el noticiero City Noticias se privilegiaron las declaraciones de la ciudadanía, la cual emitió comentarios -a pesar de la suspensión de los servicios- como una protesta útil; en el noticiero CM\& se destacaron las declaraciones de las autoridades, que expresaban el trauma que el quebrantamiento de los servicios públicos produjo en la ciudadanía. Concluyeron que, tanto el noticiero Caracol como el de RCN, emplearon el paradigma de protesta para retratar el acontecimiento, pero mientras el primero destacó los enfentamientos entre la policía y los manifestantes y los actos de violencia, el segundo destacó su marginalidad de los manifestantes y su falta de legitimidad para protestar. 


\section{Marco metodológico}

La propuesta del presente artículo se inscribe en el análisis crítico del discurso, en particular, en el estudio del framing periodístico. Esta categoría, que desde los estudios sociológicos y mediáticos se conoce como framing, en el enfoque lingüístico de Van Dijk (2003, pp. 244-245) se conoce con el nombre de esquema o superestructura, en las traducciones al español de su obra.

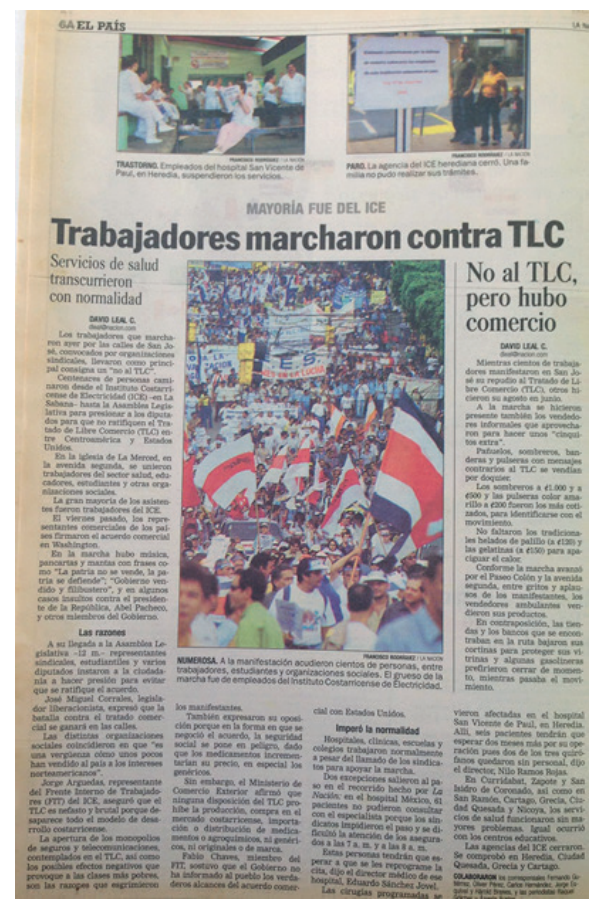

Figura 1. Noticia de D. Leal. 2004.

"Trabajadores marcharon contra el TLC". La Nación, 1/6, p. 6A.

El estudio del framing periodístico no es, estrictamente, una perspectiva lingüística, sino propiamente mediática. Pertenece a los estudios en comunicación y se encuentra vinculada a la noticia, entendida como mensaje codificado por productores profesionales periodísticos y decodificado por su público o audiencia (el estudio del framing se inscribe en el modelo comunicativo codificacióndecodificación de la semiótica estructuralista). Si bien el framing no ha sido definido en términos conceptualmente precisos, debido a las múltiples definiciones y operacionalizaciones aparecidas en el ámbito académico (Valera-Ordaz, 2016), se ha llegado a cierto consenso en el ámbito de los medios de comunicación de masas. En este sentido, en los estudios en comunicación la propuesta más utilizada es la de Entman (1993), para quien la acción encuadrar (to frame) "implica seleccionar algunos aspectos de una realidad percibida y destacarlos en un texto comunicativo, con el fin de promover una definición específica de un problema, una interpretación causal del mismo, su evaluación moral y la recomedación de una propuesta para solucionar o tratar el problema descrito" (p. 52). En la propuesta de Entman se ofrecen cuatro categorías generales de análisis. En este artículo, al final del presente apartado, se plantean con detalles una serie de categorías para analizar el framing informativo de las manifestaciones o marchas, que sin dificultad se puede incribir en las categorías generales propuestas por Entman.

Como ya dijimos, nuestro principal caso de estudio es una noticia sobre una manifestación realizada durante el ciclo de movilizaciones realizadas en Costa Rica para protestar en contra de la aprobación del Tratado de Libre Comercio (entre Estados Unidos, Centroamérica y República Dominicana), que finalmente fue aprobado por referendum el 7 de octubre del 2007 (con un apoyo de un 52\%, aproximadamente). Se trata de la noticia "Trabajadores marcharon contra TLC" $\left(L N^{3}, 2004\right.$, p. 6A), publicada en el periódico La Nación, diario de referencia dominante en Costa Rica (ver Figura 1). El movimiento en contra de la aprobación del tratado comercial con Estados Unidos pertenece a aquellas acciones colectivas que han sido investigadas, en el ámbito de la sociología de las acciones colectivas, desde el paradigma de la movilización de recursos, ya que se organizó el conflicto alrededor de un proceso comercial (frente al paradigma identitario, desde el que se estudian los movimientos feministas o LGTB). 
Nos interesa identificar, analizar e interpretar, categorías relevantes en la construcción informativa de las marchas o manifestaciones. A partir de la investigación exploratoria del corpus recogido, se han identificado procedimientos recurrentes que se pueden convertir en categorías de análisis para estudios posteriores. Es decir, formulamos una serie de categorías que, por lo general, se encuentran en el framing mediático de las manifestaciones o marchas. Su comprensión rigurosa y detenida también permite reforzar su incorporación como tópico de discusión en los cursos de análisis del discurso de las escuelas de comunicación. ¿Qué categorías puede investigar el analista de discurso cuando se acerca a la representación informativa de una manifestación? Podemos investigar:

- $\quad$ si la noticia es equilibrada o desequilibrada cuantitativa o ideológicamente en el uso de las fuentes;

- si se carnavaliza o no la apariencia externa y el comportamiento de los manifestantes;

- $\quad$ si el medio periodístico determina a la baja o al alza el número de manifestantes frente a las cifras manejadas por los organizadores y por la policía (contabilización de los manifestantes);

- $\quad$ si existe congruencia o disonancia entre el texto y la imagen de la noticia;

- $\quad$ si se muestran o no como actores que quebrantan las normas sociales (es decir, si se destacan o no como actos de violencia aislados o, por el contrario, generalizados);

- si los manifestantes quedan identificados o no como integrantes de sectores representativos de la población (representatividad);

- $\quad$ si se presentan o se omiten los objetivos de los manifestantes;

- si se destacan o no las discusiones internas de los organizadores de las manifestaciones;

- si se utilizan o no recursos de atenuación sobre la relevancia social de las manifestaciones.

Nuestra propuesta también es, en parte, multimodal (Kress y van Leeuwen, 2006), ya que buscamos identificar el sentido que surge de la interacción entre el texto y la imagen (la fotografía, por una parte, y el pie de foto, el titular y la noticia, por otra) desde la propuesta clásica, todavía vigente, de las funciones de anclaje y de relevo de Roland Barthes (1986a; 1986b), que se ha integrado en los estudios sobre la multimodalidad, en los estudios visuales y en la semiótica visual, perspectivas que cuentan con objetivos similares.

\section{Categorías de análisis en la representación informativa de las manifestaciones sociales}

\subsection{El relato informativo "equilibrado" en la representación informativa de las manifestaciones}

Idealmente, desde el punto de vista de las normas de desempeño profesional, el relato informativo de los conflictos simbólicos debe estar ideológicamente equilibrado, es decir, ser imparcial o carecer de sesgo. ${ }^{4} \mathrm{El}$ origen del relato informativamente equilibrado procede de la ideología de la objetividad, promovida por las empresas informativas, una vez que decidieron abandonar - por lo menos en sus declaraciones de intenciones- el periodismo partidista decimonónico. El relato equilibrado, como señalan Ericson, Baranek y Chan (1989, p. 217) 
pertenece al "ritual estratégico de la objetividad, en el que se ofrecen las perspectivas y se deja al lector decidir donde reside la verdad". Teniendo presente que la objetividad es una norma, la empresa informativa se define como una más entre las instituciones representativas de la democracia liberal pluralista.

Se conoce como equilibrio informativo la decisión de visibilizar en las noticias y reportajes las voces de los actores protagonistas de los procesos sociales, es decir, las perspectivas opositoras. Por ejemplo, si existen dos posiciones sobre un conflicto social -canalizado, en términos temporales, a través de la realización puntual de una manifestación-, la empresa periodística que decide emplear el equilibrio informativo como norma ofrecerá en la noticia citas o declaraciones tanto de las fuentes que han organizado la marcha como de las fuentes opositoras. Este principio, como afirman Meyer y Staggenborg (1996, p. 1641-2), suele aplicarse de manera mecanicista: se busca a los dos actores en conflicto, se entrevistan y parte de sus declaraciones aparecen en las noticias. En estos términos, el equilibrio cuantitativo es entendido por la empresa periodística como equilibrio ideológico. ¿Qué marca textual identificaría el equilibrio ideológico? La presencia de un número idéntico de citas de los dos actores en conflicto. Desde el punto de vista de las empresas periodísticas, este equilibrio cuantitativo implicaría equilibrio ideológico, por cuanto se otorga acceso a las dos voces en conflicto.

De entre todas las categorías de análisis que se pueden incorporar en la metodología de estudio de la noticia de una manifestación, la del equilibrio informativo es la más problemática a la hora de quedar operacionalizada. La imparcialidad del relato ideológicamente equilibrado constituye una pretensión incumplida, como todo proyecto perteneciente a la ideología de la objetividad. Por una parte, Ericson, Baranek y Chan (1991, p. 186) destacan que

la frecuencia con la que una fuente es citada no indica nada sobre su representación. Algunas fuentes aparecen relativamente en frecuentes oportunidades, pero no son representadas favorablemente, y quedan preferiblemente retratadas como actores desviados cuya autoridad está siendo desacreditada por los medios informativos en conjunción con las fuentes preferidas

Asimismo, Stevenson y Green (1980, p. 116) señalan las dificultades de determinar el grado de sesgo, ya que si un medio trata equilibradamente, en términos cuantitativos, las voces de dos actores que tienen distinto poder social, será acusado de ofrecer una imagen sesgada -distorsionada- de la realidad, mientras que si incorpora en las noticias las diferencias socialmente existentes entre los dos actores, al otorgar más espacio a una de las fuentes (la que cuenta con mayor poder), la empresa informativa será acusada de ofrecer una visibilidad informativa desequilibrada de los actores sociales y, en consecuencia, sesgada. Nos enfrentamos al viejo dilema, también clásico en el estudio de los estereotipos o imágenes de los grupos sociales, de representar la estructura social (por ende, desequilibrada) o de representar un proyecto de sociedad ideal y, por lo tanto, justo y equilibrado (pero alejado de la realidad social presente). Entre los investigadores, las posiciones positivistas respaldan la primera posición: se argumenta en estos casos que, si uno de los grupos sociales es muy minoritario, no debe de adquirir - porcentualmente hablando- la misma representación -en las noticias sobre los conflictos sociales- que grupos con mayor poder o participación social, ya que, si no fuera así, se estaría sobrerrepresentando al primer grupo. Posiciones postmodernas respaldan la segunda: se argumenta que no se pueden reproducir las diferencias de poder existentes en la sociedad en el discurso mediático porque, en este caso, los grupos minoritarios siempre carecerían de representación y que, por el contrario, los medios deben actuar desde posiciones de defensoría 
de estos grupos, al contribuir al proceso democrático, aunque queden sobrerrepresentados desde un enfoque realista (esta defensoría se convertiría en una acción afirmativa propugnada por las empresas periodísticas).

Surgen otros problemas metodológicos a la hora de definir el relato informativo ideológicamente equilibrado. Si uno de los actores de un movimiento social está conformado por una coalición: ¿qué voz, en el seno de esta coalición debe constituirse -en el relato informativo equilibrado entre perspectivas opositoras-, como el actor más representativo? En los relatos informativos no suelen tener acceso, como fuentes, todos los actores de una coalición. Además, más allá de la dificultad metodológica de definir el equilibrio informativo, aunque la empresa periodística quisiera aplicar una definición precisa del mismo, distintos factores empresariales dificultarían su ejecución, el más importante de ellos el compromiso de las empresas informativas con su audiencia y con la estructura de poder político y económico de la sociedad en la que actúan.

Consideramos que -con propósitos operativos- se puede ampliar o estrechar la semántica de lo que entendemos por relato ideológicamente equilibrado y hablar, hasta cierto punto, de su presencia en el discurso informativo. Es desaconsejable, en todo caso, proponer una definición restringida. Consideramos que se puede ofrecer una visión lo más abarcadora posible del equilibrio ideológico, para que no sea solo identificado en términos cuantitativos: es aquella noticia que ofrece la mayor diversidad posible de reivindicaciones de los dos lados de un conflicto social y, si cada lado se encuentra integrado por una coalición de actores, aquella noticia que visibilice la mayor diversidad posible de reivindicaciones en el seno de una coalición. En otras palabras, es aquella noticia ideológicamente plural o, en términos bajtinianos, es aquella noticia que exprese, en el marco de la visibilidad de las fuentes, una visión dialógica de los conflictos sociales. En estos términos, estamos de acuerdo con Lozano (1994, p. 144) al señalar que para "los interesados en evaluar qué tan balanceado es el acceso proporcionado por los medios informativos a diferentes actores, grupos y organizaciones, sean políticas o no, conviene definir como unidad de análisis a la fuente informativa y no al artículo o nota".

Si nos acercamos a nuestro corpus de estudio, la noticia "Trabajadores marcharon contra TLC” (LN, 2004, p. 6A) ¿expresaría esta última concepción del equilibrio informativo? En esta noticia aparecen dos fuentes de actores opositores a la aprobación del Tratado, frente a una fuente a favor. En contra, se encuentran Jorge Arguedas, del Frente Interno de Trabajadores (FIT) del ICE, y José Miguel Corrales, diputado liberacionista (uno de los pocos que asumieron esta posición en el seno de este partido político). A favor quedó visibilizado el Ministerio de Comercio Exterior (fuente estrictamente institucional, ya que no se trata de un sujeto humano que ostenta un rol específico dentro de una organización). Pero más importante -de cara a comprender el punto de vista asumido por las fuentes- es conocer el tópico del que hablan las fuentes y la perspectiva desde la que lo hacen. En este sentido, José Miguel Corrales, en aquel entonces diputado del PLN (a contracorriente de la posición mayoritaria de su partido), "expresó que la batalla contra el tratado comercial se ganará en las calles." (LN, 2004, p. 6A). De manera bastante imprecisa, sin detallar su procedencia, el medio de comunicación también otorga $\mathrm{VOz}$-en estilo directo- a distintas organizaciones sociales en contra del TLC, quienes definieron como "una vergüenza cómo unos pocos han vendido al país a los intereses norteamericanos". Por último, Jorge Arguedas, del Frente Interno de Trabajadores (FIT) del ICE, "aseguró que el TLC es nefasto y brutal porque desaparece todo el modelo de desarrollo costarricense" (LN, 2004, p. 6A). Se visibiliza su discurso en estilo indirecto. 
La fuente a favor del TLC es institucional y, además, se ofrece en estilo indirecto. El periódico la sitúa, en la noticia, después de las declaraciones de los actores en contra de la aprobación del Tratado. Con el empleo del conector "Sin embargo", el discurso periodístico construye una refutación simulada. La fuente a favor del TLC parece estar refutando directamente a las fuentes en su contra: "Sin embargo, el Ministerio de Comercio Exterior afirmó que ninguna disposición del TLC prohíbe la producción, compra en el mercado costarricense, importación o distribución de medicamentos o agroquímicos, ni genéricos, ni originales o de marca" ( $L N, 2004$, p. 6A). De las tres fuentes en contra del TLC, solo una comunica las razones para oponerse a su aprobación, las consecuencias negativas que traerá para la economía. Se trata, en todo caso, de una proyección futura muy general ("la desaparición del modelo de desarrollo costarricense"). Las otras dos fuentes no comunican los motivos de su oposición al TLC: se trata de una imprecación a los actores opositores y de una proyección a corto plazo de una victoria del No al TLC. En cambio, la fuente que se elije a favor del TLC para quedar visibilizada en el relato informativo ofrece causas razonadas para abogar en favor de la aprobación del Tratado. Podemos comprobar, en consecuencia que, si bien nos enfrentamos a una noticia con un desequilibrio cuantitativo en favor de los actores en contra del Tratado, se trata de un relato informativo ideológicamente orientado, en términos de las fuentes visibilizadas, a favor de su aprobación, ya que estas últimas quedan retratadas como los únicos actores que cuentan con argumentos razonados para defender su posición en el espacio público de discusión.

\subsection{Las acciones de los manifestantes como ocasiones festivas}

En ocasiones, en las noticias de las manifestaciones el interés se centra en mostrar, a nivel de la representación fotográfica, los disfraces de algunos manifestantes, frente a las pancartas reivindicativas. En lugar de escoger las fotografías que muestran, en plano general o panorámico, el 'río' de manifestantes en las avenidas, los centenares o miles de personas que pudieron haber participado en las manifestaciones, se escoge la fotografía que muestra a un grupo de activistas disfrazados. No son fotografías que traten de verificar la magnitud numérica de participantes alcanzada por los organizadores de la manifestación.

En las páginas de los periódicos hegemónicos, las fotografías de las manifestaciones organizadas por el sindicalismo también se centran, en algunos casos, en mostrar acciones festivas, en lugar de mostrar las pancartas y los lemas reivindicativos. En la fotografía de la noticia "Indisposición general para participar en bloqueos" (Villalobos, 13 de marzo de 2007) se ofrece en primer plano a manifestantes que tocan la trompeta y llevan la máscara de Scary Movie. En el texto de la noticia "Marcha pacífica contra TLC" (Villegas, 18 de noviembre de 2005) se declara: "La marcha fue colorida pues muchos participantes usaron disfraces con mensaje contra el TLC, hubo música y hasta comparsa”. Sólo después se abre un apartado dentro de la noticia- en el que visibiliza el mensaje de los manifestantes.

En ocasiones, el medio de comunicación llega a crear una noticia secundaria, dependiente de la principal, para describir el desvío 'festivo' de los objetivos inicialmente planteados. La noticia secundaria "No al TLC, pero hubo comercio", de la noticia principal “Trabajadores marcharon contra el TLC" (Leal, 1 de junio de 2004), se dedica a detallar la venta de pañuelos, sombreros, banderas y pulseras por parte de los vendedores ambulantes. En este caso, el medio periodístico se encarga de construir, mediante el titular de esta noticia secundaria, una aparente contradicción entre los manifestantes. Esta noticia secundaria se 
refiere al auge de las ventas ambulantes durante la realización de la manifestación. Mediante el titular "No al TLC, pero hubo comercio", el periódico se encarga de atribuir una conduca contradictoria y paradójica entre los opositores al TLC, que por una parte se opondrían al comercio (internacional), mientras que, por otra parte, fomentarían el comercio minorista de las ventas callejeras.

Una representación informativamente irresponsable mostrará exclusivamente las acciones "carnavalescas" frente a los objetivos de los manifestantes. Estos últimos -teniendo en mente parcialmente que los medios de comunicación tienen la tendencia a destacar los aislados actos de violencia de las marchas-, suelen convertir el desarrollo de estas últimas en actos tanto festivos como reivindicativos. El problema, en términos de representación informativa, radica en la resignificación ideológica que algunos medios de comunicación realizan de las manifestaciones como actos exclusivamente carnavalescos; en estos casos, los mass media expresan, explícita o implícitamente, que los manifestantes carecen de "verdaderos" objetivos reivindicativos.

\subsection{Número de manifestantes}

Tanto para los actores organizadores como para los opositores, la efectividad de una manifestación suele plantearse en términos de la cantidad de participantes que llegaron a ser movilizados: se dice que una marcha fracasó o tuvo éxito según el número de activistas y simpatizantes contabilizados en el acontecimiento. Una vez realizada la manifestación, parte importante de la controversia que protagonizan los actores sociales gira alrededor del número de activistas que protestaron en las calles. En las noticias, por lo general, los periodistas incorporan dos cifras, la proporcionada por los organizadores de la marcha, que los actores opositores consideran "inflada" al alza, y la proporcionada por la policía, que los convocantes consideran que responde a la baja. Las "estimaciones" no sólo forman parte de la representación informativa de las manifestaciones, sino también de relatos sobre desastres naturales (estimaciones del número de víctimas) o conflictos bélicos (estimaciones del número de muertos o de desplazados).

Por lo general, los medios informativos -entre ellos, las agencias de prensa- ofrecen una cifra de participantes que se encuentra a medio camino ente las apreciaciones de los organizadores de la marcha y las fuentes policiales. Un ejemplo lo representa la noticia sobre el acontecer político español, publicada en la sección "Internacionales", "Podemos demuestra músculo político con marcha masiva" (AFP, 2 de febrero de 2015), proporcionada por la agencia AFP. En el desarrollo de la noticia, se ofrece primero las cifras de los actores organizadores y de la policía: "Unas 100.00 personas, según la Policía, y 300.000, según los convocantes, ocuparon el centro de Madrid para escuchar a Iglesias". Previamente, en el primer párrafo, para responder al quién -que forma parte de las 5W's de la prototípica pirámide invertida- la agencia de prensa expresa sus propias estimaciones: "El partido antiliberal español Podemos congregó al sábado a más de 100.000 personas en Madrid”. Se trata de una apreciación más cercana a las cifras policiales que las aportadas por los organizadores de la manifestación, orientación que se suele dar en la representación informativa de estos acontecimientos por parte de los medios hegemónicos.

En otro ejemplo, procedente de la noticia titulada "Una multitudinaria marcha celebra en Madrid la igualdad de derechos para los homosexuales", el diaro español El País ofrece estimaciones - procedentes del mismo diario- situadas entre las cifras ofrecidas por los organizadores y las aportadas por la policía, aunque, como en el caso anterior, son más 
cercanas a estas últimas: "Más de 250.000 personas según cálculos de este periódico (dos millones según los organizadores y 97.000 de acuerdo con la policía) se manifestaron con motivo del Día del Orgullo Gay" (De Benito, 3 de julio de 2005, párr. 2).

Situándonos en el conflicto originado a partir del debate sobre la aprobación del TLC con Estados Unidos en Costa Rica, La Nación no ofreció, casi nunca, estimaciones propias sobre los participantes convocados, o estimaciones aportadas por los organizadores o la policía. En ocasiones, como en la noticia "Marcha contra el TLC termina sin incidentes" (Oviedo, 26 de febrero de 2007), una de las más multitudinarias, el medio de comunicación no se ocupó en ningún momento (ni en el titular, el pie de foto o el texto central) de mencionar el número aproximado de manifestantes. Esta empresa periodística, por lo general, utilizó el impreciso "centenares" o "miles". Así ocurrió también en el titular "Trabajadores marcharon contra el TLC" (Leal, 2004, p. 6A), en el cuerpo de la noticia: "Centenares de personas caminaron desde el Instituto Costarricense de Electricidad (ICE) - en La Sabana- hasta la asamblea legislativa". Es más, La Nación optó, en una ocasión, por incorporar -en lugar de una cifra aproximada de manifestantes- el número de metros de vía que ocuparon los participantes, como en el titular "Marcha llenó 200 metros del Paseo Colón" (Villegas y Herrera, 21 de octubre de 2003). Como medida excepcional, encargó la realización de un "conteo científico" en la manifestación del 27 de febrero de 2007, cuyo resultado fue destacado en el titular: "Marcha pacífica reunió a 23.500 opositores al TLC" (Oviedo, 27 de febrero de 2007).

\subsection{Relaciones entre la imagen y el texto del relato informativo}

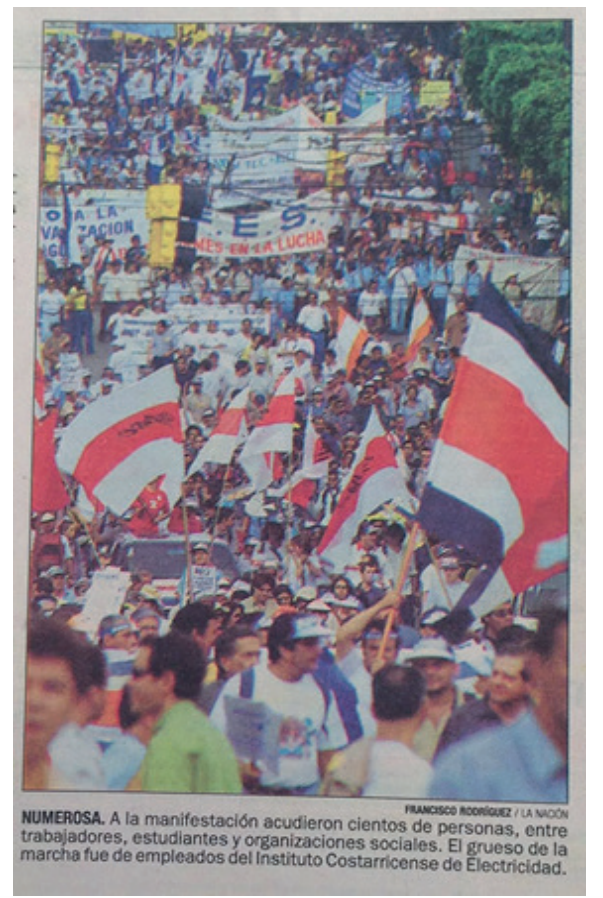

Figura 2. Fotografía y pie de foto de la noticia de D. Leal. 2004. "Trabajadores marcharon contra el TCL". La Nación, 1/6, p. 6A.
Distintos tipos de relaciones se pueden dar en la yuxtaposición entre la imagen y el texto del relato informativo, en particular, el pie de la fotografía: (a) el texto puede amplificar los significados connotativos de la foto; (b) puede producir o inventar un significado connotativo nuevo que se proyecta retroactivamente sobre la imagen; y (c) puede contradecir la imagen, con lo que se produce un significado connotativo compensatorio (Barthes, 1986a, p. 23). En muchos "pies de fotos" incorporadas en las noticias de manifestaciones, la empresa periodística produce este último significado connotativo: mientras que en la imagen se observan miles de manifestantes, en el texto del pie de foto afirma que la participación se reduce a cientos de activistas.

Sobre la primera función, en la yuxtaposición foto-pie de foto de los relatos informativos, la relación que se establece no es de relevo, sino de anclaje (ver ambas funciones en Barthes, 1986b), debido a la necesidad de fijar la ambigüedad de la imagen. En este sentido, el lector tenderá a elaborar el significado final partiendo, sobre todo, del texto: la lectura de la 
imagen es más ambigua, mientras que los signos verbales ("cientos" o "miles") tienen mayor valor designativo, referencial.

Sobre la tercera función, la contradicción entre la fotografía de la manifestación y el pie de foto no es común, pero puede llegar a darse. En la fotografía se observan centenares de manifestantes, mientras que en el pie de foto se habla de "decenas de sindicalistas" (Leal, 1 de junio de 2004). En este último caso, además, el empleado público queda reducido al papel de sindicalista, con el fin de fortalecer el vínculo entre la participación en protestas públicas y el sindicalismo. La palabra - pie de foto- llega a contradecir a la imagen hasta producir una connotación compensatoria.

En la representación fotográfica de la manifestación realizada el 31 de mayo de 2004, el reportero gráfico, en el lugar del hecho, realizó un trabajo de focalización, de encuadre. En la manifestación participaron miles de personas, pero el reportero fotografió cientos de participantes. La fotografía fue tomada a nivel del suelo, aunque el fotógrafo aprovechó una elevación del terreno para captar una cantidad de manifestantes, que marchaban detrás, que hubiera sido difícil captar si hubiera sido totalmente plano. Es de esperar que el reportero gráfico realizara diversas tomas y que el equipo editorial eligiera esta fotografía, por diversas razones, para que se publicara finalmente. En este caso, la fotografía muestra centenares de manifestantes, y también se emplea esta cifra en el pie de foto (Figura 2).

Recordemos que, hipotéticamente, y a partir de una misma fotografía que muestra un "río de manifestantes" en una carretera, el periodista, en el marco de la función de anclaje que quiere proyectar a la imagen, puede incorporar dos o más "pies de foto" diferentes (es decir, con enunciados distintos), que incidirán en la atribución del significado final de la imagen por parte de los lectores. A partir de la misma fotografía (el grupo compacto de manifestantes en la calle), se puede incorporar el hipotético pie de foto "Manifestantes defienden sus derechos sociales", con lo que se destacan o focalizan, en el acontecimiento, las causas que lo han provocado, mientras que el pie de foto "Manifestantes cortan la carretera e impiden a miles de personas llegar al trabajo" focaliza el acontecimiento en las consecuencias que tiene para los actores sociales - la ciudadanía- que no lo han organizado, aunque no se muestren en la imagen. En resumen, la fotografía de la manifestación es la misma, pero los 'pies de foto' incorporados son ideológicamente opuestos. Lo mismo ocurre cuando se inserta una misma foto en una noticia con titulares ideológicamente opuestos.

\subsection{Representación de acciones "desviadas" de las normas sociales: las marchas o manifestaciones como causantes de desórdenes y disturbios}

Esta última ocurre si se describe a los activistas como actores que quebrantan las normas sociales (es decir, si se destaca la violencia y los ataques de los manifestantes contra la policía o actores opositores, el corte de carreteras y las consecuencias que acarrean para los automovilistas, o el cierre de los servicios públicos de salud y las consecuencias negativas que acarrean a los usuarios). López del Ramo y Juan Francisco Torregrosa (2011), en su análisis de la representación informativa de huelga general del 2010 en España, consideraron cuatro tipos de bloqueos: (a) de transportes, (b) de mercados, (c) de actividades laborales y (d) de centros públicos. Recordemos que la desviación de las normas sociales es un claro predictor de la noticiabilidad de un acontecimiento (Shoemaker, Chang y Brendlinger, 1987) y que cualquier acto violento aislado, en una manifestación pacífica, "disparará" automáticamente 


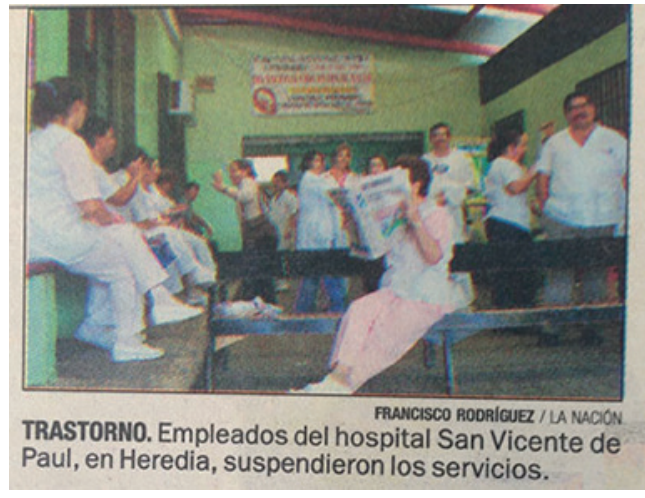

Figura 3. Fotografía y pie de foto de la noticia de D. Leal. 2004. "Trabajadores marcharon contra el TCL". La Nación, 1/6, p. 6A. la noticiabilidad de esta última. Se acaba por convertir en un símbolo -en una sinécdoque generalizante- que termina por caracterizar, ante la sociedad, a todos los manifestantes y a la definición misma de la acción colectiva a lo largo del tiempo. Se presupone que el quiebre de la cotidianedidad es una consecuencia directa e inevitable de toda manifestación, de ahí que, desde la misma convocatoria de una marcha, el Gobierno apele a la menor interrupción posible de los servicios públicos y del horario laboral. Por su parte, Van Dijk (2003, pp. 263-276) analizó la construcción de los "disturbios" ocurridos en Gran Bretaña en 1985 por la población negra de origen

antillano; los medios destacaron los actos violentos como procedentes del comportamiento patológico de los manifestantes (drogas, falta de adaptación) en lugar del desempleo o la discrminación.

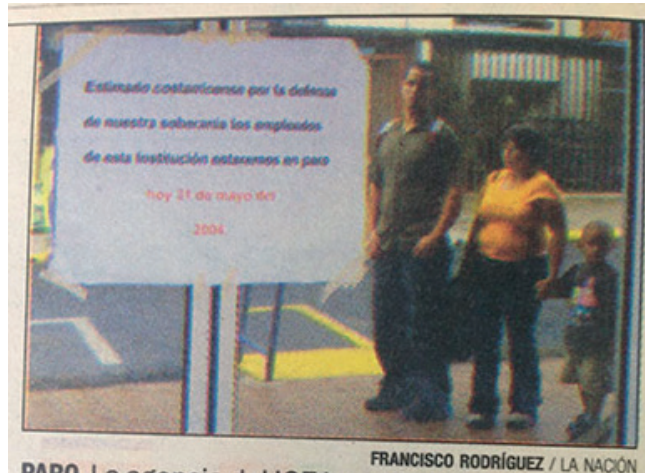

PARO. La agencia del ICE herediana RODRIGUEZ / LA NACON milia no pudo realizar sus trámites.

Figura 4. Fotografía y pie de foto de la noticia de D. Leal. 2004. "Trabajadores marcharon contra el TCL'. La Nación, 1/6, p. 6A.
Los titulares pueden destacar la (escasa o generalizada) interrupción de la cotidianeidad, como en "Marcha tuvo poca afectación en servicios públicos" (Fallas, 26 de febrero de 2007), tema que también se puede ofrecer en el pie de foto de dos fotografías: "Trastorno. Empleados del hospital San Vicente de Paul, en Heredia, suspendieron los servicios" y "Paro.Laagencia del ICE herediana cerró. Una familia no pudo realizar sus trámites" (Leal, 1 de junio de 2004, p. 6A) (Figuras 3 y 4). En alguna ocasión, se construye como noticia independiente este quebrantamiento de la cotidianeidad laboral, sin que forme parte de la noticia de la manifestación propiamente dicha: "Miles de estudiantes sin clases

por marcha, pero apoyo de docentes a movimiento es parcial" (Oviedo, 26 de febrero de 2007). La marcha se convierte en un acontecimiento absurdo: obtuvo escasa convocatoria, pero las consecuencias directas en el quebrantamiento de la cotidianeidad fueron enormes.

El medio de comunicación puede, por implicatura, orientar la comprensión que tienen los lectores de las manifestaciones como acciones "por esencia” violentas. Esta connotación se obtiene de volantas 4 como "Imperó la normalidad", de la noticia "Trabajadores marcharon contra TLC" (Leal, 1 de junio de 2004) o de "En el 2006 la marcha fue pacífica" o "Vigilancia de cargo de 350 policías", de la noticia "Marcha saldrá del Parque Central y recorrerá 9 cuadras" (Carranza, 1 de mayo de 2007), o de "Manifestación pacífica ayer en Paseo Colón", de la noticia "El NO evoca a próceres para pedir fusilar TLC en las urnas" (Murillo y Agüero, 1 de octubre de 2007). También se obtiene esta implicatura de titulares como "Marcha pacífica reunió a 23.500 opositores al TLC" (Oviedo, 27 de febrero de 2007), "Pacífica marcha contra el TLC" (Oviedo, 24 de octubre de 2006), "Frente promete marcha pacífica hoy" (Fallas, 26 de febrero de 2007), 
"Arias confía en una marcha sin violencia" (Umaña, 23 de febrero de 2007), "Marcha contra el TLC termina sin incidentes" (Oviedo, 26 de febrero de 2007) o "Marcha tuvo poca afectación en servicios públicos" (Fallas, 26 de febrero de 2007). Por implicatura, los actores sociales que se oponen a la marcha y el medio de comunicación consideran que la ausencia de actos de violencia, en las manifestaciones, es una situación de excepcionalidad y no de normalidad. A nivel connotativo, se infiere que las manifestaciones se enfrentan al equilibrio social.

Los actos violentos aislados suelen ser etiquetados por los Gobiernos o por los medios de comunicación como comportamientos regulares de los manifestantes. En alguna ocasión, y como consecuencia de la dependencia que tienen los medios de comunicación de los subsidios informativos ofrecidos por los Gobiernos, incluso la violencia potencial de las manifestaciones -que reactivan los miedos y las alarmas sociales que ya desencadenó, en el siglo XIX, el concepto de "masa"- se convierte en valor noticiable. Los organizadores de las manifestaciones ante el TLC, a la defensiva, ante las acusaciones de violencia, necesitaron destacar una imagen de defensores del orden público.

\subsection{Representatividad social de los manifestantes}

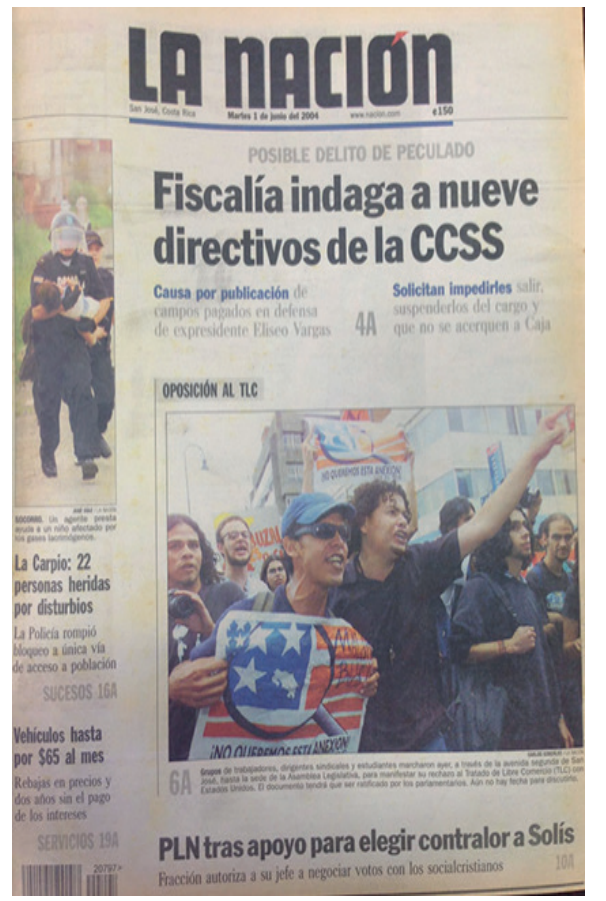

Figura 5. Fotografía de portada "Oposición al TLC" de la noticia "Trabajadores marcharon contra del TLC" de Leal, D. (1 de junio de 2004). La Nación.

Las noticias de manifestaciones también se encargan de construir discursivamente la representatividad de los participantes. Cuando los intereses ideológicos del movimiento social chocan contra la ideología implícita del medio de comunicación, quedan perfilados los manifestantes como integrantes de grupos de interés que defienden reivindicaciones muy específicas $\mathrm{y}$, en ocasiones, en conflicto con los intereses generales. Es lo que ocurre en la noticia "Trabajadores marcharon contra el TCL" (Leal, 1 de junio de 2004). En la volanta se declara "Mayoría fue del ICE". Asimismo, en el cuerpo de la noticia se afirma que la mayor parte de los participantes fueron trabajadores del ICE: "La gran mayoría de los asistentes fueron trabajadores del ICE", a los que se unieron trabajadores del área de la salud, educadores, estudiantes e integrantes de otras organizaciones sociales (Leal, 1 de junio de 2004, p. 6A). Este trabajo de focalización, consistente en identificar a los manifestantes con sectores muy específicos, también se realizó en la fotografía de la marcha empleada en la portada o primera página. En lugar de mostrarse el "río" de manifestantes que llenan la avenida de izquierda a derecha, en la fotorafía de la portada aparece un grupo pequeño de manifestantes, que a través de una lectura semiótica se pueden identificar como estudiantes (Figura 5). Con esto, se realiza una táctica de invisibilización visual -por lo menos en la portada, la página más relevante del periódico- de los demás grupos sociales participantes en la marcha. No nos encontramos ante la atenuación 
de la importante participación del movimiento estudiantil en la marcha, sino la exclusión visual de los demás sectores participantes. Otros titulares del ciclo de protestas contra el TLC nos indican la sectorialización de las marchas - de sus organizadores y participantes- realizada por la empresa periodística, como los sindicalistas: "Sindicatos en pulso hoy contra TLC" (Villalobos, 20 de octubre de 2003) o los educadores: "Débil apoyo de empleados públicos a paro nacional" (Villegas y Ávalos, 27 de agosto de 2004).

Es común la focalización visual de las demostraciones en grupos pequeños, sobre todo cuando se enfrentan a la policía, ya sea proactiva o reactivamente. En la representación informativa de una manifestación británica realizada contra una nueva ley de criminalidad (British Crminal Justice Bill), Hansen et al. (1998, pp. 208-213) explican que, mientras el Daily Mail ofreció una fotografía de tres manifestantes que se enfrentan directamente con la policía, el periódico The Guardian mostró a dos manifestantes que descansan en el piso con las parcartas bajas con bebidas al lado, mientras la policía se encuentra detrás -en pie- en actitud expectante. En ambas fotos quedan visualizados la policía y un grupo de manifestantes, pero la distinta yuxtaposición y asociación que un actor tiene respecto del otro produce distintas connotaciones. En la primera foto, el grupo de manifestantes queda revestido del atributo del comportamiento violento, mientras que en la segunda queda caracterizado como acto políticamente motivado, pero desorientado y desmotivado en su activismo.

\subsection{Visibilización u ocultamiento de los objetivos de los manifestantes}

Los actores sociales reivindican la legitimidad de sus objetivos y, para lograrlo, organizan y participan en manifestaciones. Pero no siempre la empresa periodística muestra, en la noticia de una manifestación, los objetivos de los organizadores. El silencio es semiótico; es decir, significa. Entre las excepciones se encontraron "Trabajadores marcharon contra el TCL" (Leal, 1 de junio de 2004), "Pacífica marcha contra el TLC" (Oviedo, 24 de octubre de 2006), "Gremios marcharán contra TLC" (Loaiza, 13 de octubre de 2003), "Sindicatos en pulso hoy contra TLC" (Villalobos, 20 de octubre de 2003) o "Trabajadores marcharon por TLC y referendo" (Leal, 2 de mayo de 2007), aunque en este último caso no se especifica en el titular que se trata de una marcha en contra y no a favor del TLC, como parece indicar la preposición "por".

\subsection{Otras categorías: visibilidad del disenso interno de los manifestantes y recursos léxicos de atenuación de la relevancia de las manifestaciones}

En cada actor reivindicador pueden desarrollarse orientaciones rivales. Sin embargo, la existencia de dos posturas mayores en conflicto suele constituir una de las características prototípicas del relato informativo del conflicto. Se simplificará en estas ocasiones. Además, la existencia de redes de coaliciones tiende a quedar invisibilizada en las representaciones informativas. Como afirma Knight (1982, p. 18), "la polarización de los sujetos informativos en dos partes presupone que no existe una multiplicidad de posiciones e intereses que, fluidas e intercambiables, interactúan y sobreimponen". En todo caso, si la coalición "amenaza" el status quo con sus reivindicaciones, los medios de comunicación hegemónicos tenderán a visibilizar el disenso interno que se produzca en su interior. No se dio en el caso de la representación informativa de las marchas contra el TLC, que estuvieron organizadas por una coalición de actores que definieron objetivos altamente consensuados. 
Por último, no podemos dejar de mencionar un procedimiento que, aunque se da en pocas oportunidades, es pertinente a la hora de considerarlo como categoría de análisis. La trivialización, ridiculización o demonización de las manifestaciones o del lenguaje de los manifestantes mediante la utilización de distintas elecciones léxicas, más allá de la elección de fuentes, focalizaciones (inclusiones, exclusiones) y frames. La voz periodística puede utilizar sustantivos atenuadores que contribuyen a otorgar menor relevancia social al acontecimiento. Por ejemplo, en la volanta de la noticia "Gremios marcharán contra TLC" (Loaiza, 13 de octubre de 2003) se emplea el término 'caminata' para referirse a la marcha hacia el congreso del 20 de octubre.

\section{Conclusiones}

Las noticias de manifestaciones sociales constituyen un formato informativo por derecho propio, repetido y legitimado a través de la rutina periodística. Es decir, son noticias caracterizadas por procedimientos discursivos prototípicos. Representan un formato informativo específico, entendiendo por este último, si seguimos a Altheide y Snow (1988, p. 199), como el conjunto de reglas y lógicas que transforman y construyen las informaciones (los contenidos), normas transmitidas en estructuras y formas reconocibles.

Las noticias sobre manifestaciones cuentan con unos procedimientos discursivos verbales y visuales prototípicos que nos hemos encargado de analizar e interpretar en el presente artículo. Como hemos indicado a lo largo del artículo, podemos investigar si el medio informativo procede a: (a) omitir o presentar los objetivos de los manifestantes; (b) a trivializar o no la importancia de la manifestación o del discurso de los manifestantes; (c) a carnavalizar o no la apariencia externa y el comportamiento de los manifestantes; (d) a determinar a la baja o al alza el número de manifestantes frente a las cifras manejadas por los organizadores y por la policía; (e) a destacar o no las discusiones internas de los organizadores de la manifestación; (f) a mostrar o no a los activistas como actores que quebrantan las normas sociales (es decir, si se destacan o no actos aislados o generalizados de violencia); (g) a ofrecer una noticia equilibrada o desequilibrada cuantitativamente o ideológicamente en el uso de las fuentes; (h) a omitir o no los objetivos de los manifestantes; y, finalmente, (i) a identificar o no a los manifestantes como integrantes de sectores representativos de la población. Según los casos, el medio periodístico, en distintos grados, elaborará un framing positivo o negativo sobre las reivindicaciones planteadas.

Algunos relatos informativos sobre manifestaciones no representan marchas ya realizadas, sino por realizar en un futuro próximo. La programación o convocatoria de la manifestación se convierte en noticia, como ocurre en "Gremios marcharán contra TLC" (Loaiza, 13 de octubre de 2003), "La APSE luchará contra TLC" (Golcher, 14 de agosto de 2004), "Sindicatos anuncian paros y marchas" (Mora, 20 de noviembre de 2007) o "Grupos del 'no' planean más manifestaciones” (Fallas, 22 de julio de 2007). Pero no sólo puede adquirir noticiabilidad la convocatoria de una manifestación, o la difusión de información movilizadora que permitirá a los potenciales activistas participar en la protesta, sino también las reacciones en contra a la convocatoria mencionada: en la volanta del titular de "Gobierno advierte a quienes marchen contra TLC el lunes" (Alvarado, 26 de mayo de 2004) se afirma: "No pagará a quien no labore". Es decir, antes de la realización de una manifestación se producen diversos acontecimientos discursivos (convocatoria, reacciones opositoras de la convocatoria) que la convierten en un acontecimiento noticiable. Si la realización de la marcha es temporalmente 
próxima, pueden publicarse noticias que ofrecen lo que se denomina información movilizadora: indican en qué momento tendrá lugar la marcha (fecha, hora de inicio y de finalización), así como el trayecto que recorrerá. Es el caso de la noticia "Marcha saldrá del Parque Central y recorrerá 9 cuadras" (Carranza, 1 de mayo de 2007) o de "Sindicatos en pulso hoy contra TLC" (Villalobos, 20 de octubre de 2003), dedicada en su mayor parte a ofrecer información movilizadora sobre la marcha que, horas después, tuvo lugar ese mismo día.

\section{Notas}

1. El análisis se ejecutó en televisión, revistas informativas semanales, caricaturas y columnas de opinión.

2. En el análisis, aplicado a una muestra informativa, se destaca una evolución en los marcos interpretativos temáticos visibilizados: el antifeminismo se incorporó en los años sesenta y las divisiones entre las feministas en los setentas; por otra parte, mientras que la preocupación principal en los años cincuenta respondió a los papeles tradicionales de género y en los sesenta a lo económico y los puestos de trabajo ocupados por mujeres, en los setentas, ochentas y noventas pasa a ocupar el primer lugar el marco temático del feminismo (Terkildsen y Schnell, 1997, p. 885).

3. A partir de este momento, toda referencia bibliográfica dentro del texto principal del periódico $L a$ Nación aparecerá como LN.

4. Stevenson y Green (1980, p. 115-6) señalan que la mayoría de los estudios sobre el sesgo informativo utilizan dos definiciones comunes: la menos utilizada, escasamente útil como definición operativa, equipara al sesgo con la inexactitud y ocurre cuando se cita equivocadamente a una persona o se distorsiona su posición (generalmente se conoce el sesgo al comparar el relato con la fuente escrita que le sirvió de precedente o al encuestar a las fuentes acerca de potenciales inexactitudes en los relatos informativos); por otra parte, la definición más rigurosa equipara al sesgo con la tratamiento sistemáticamente diferente que recibe un actor o una visión de un asunto durante un extenso periodo de tiempo.

5. La volanta es el subtítulo situado encima del titular propiamente dicho. Ver Cuvardic García y Vargas Castro, 2010, p. 208.

\section{Bibliografía}

Acosta, G. L. (2013). Deslegitimación en el discurso político: un análisis de las declaraciones oficiales y de sus réplicas frente al movimiento social y político 'Marcha Patriótica'. Discurso y Sociedad. 7 (2), 364-391.

AFP. (2 de febrero de 2015). Podemos demuestra músculo político con marcha masiva. La Nación, 29A.

Altheide, D. L. y Snow, R. P. (1988). Toward a Theory of Mediation. En J. A. Anderson (Ed.). Communication Yearbook 11. (pp. 194-223). Newbury Park: Sage.

Alvarado, E. (26 de mayo de 2004). Gobierno advierte a quienes marchen contra TLC el lunes. La Nación. 26/5. Recuperado de http://wvw.nacion.com/ln_ee/2004/mayo/26/ pais5.html

Artavia, S. (5 de octubre de 2007). Arte y música cerraron campañas del SÍ y el NO. La Nación. 5/10. Recuperado de http://wvw.nacion.com/ln_ee/2007/octubre/05/pais1265518.html_

Ashley, L. y Olson, B. (1998). Constructing reality: print media's framing of the women's movement, 1966 to 1986. Journalism Quarterly. 75 (2), 263-77. 
Associated Press. (1 de octubre de 2007). Arias reitera que Costa Rica abandonará TLC con EE.UU. si no resulta. La Nación. 1/10. Recuperado de http://wvw.nacion.com/ In_ee/2007/octubre/01/pais1261332.html

Barthes, R. (1986a). El mensaje fotográfico. Lo obvio y lo obtuso. (pp. 11-27). Barcelona: Paidós. Barthes, R. (1986b). Retórica de la imagen. Lo obvio y lo obtuso. (pp. 29-47). Barcelona: Paidós.

Carranza, P. (1 de mayo de 2007). Marcha saldrá del Parque Central y recorrerá 9 cuadras. La Nación. Recuperado de http://www.nacion.com/nacional/Marcha-parque-centralrecorrera-cuadras_0_900510021.html

Castillo-Esparcia, A., García-Ponce, D. y Smolak Lozano, E. (2013). Movimientos sociales y estrategias de comunicación. El caso del 15M y de Occupy Wall Street. Estudios sobre el mensaje periodístico. 19 (1), 71-89.

Cuvardic García, D. (2002). Los marcos interpretativos textuales: herramienta metodológica para el análisis del discurso periodístico. Revista de Ciencias Sociales (Universidad de Costa Rica). 96, 83-98.

Cuvardic García, D. (2003). Relações ente meios de comunicação e movimientos sociais. Líbero. 12, 20-29.

Cuvardic García, D. (2004-2005). Construcción y representación fotográfica de los conflictos sociopolíticos: la 'huelga de educadores' del 2003 en el diario La Nación. Revista de Ciencias Sociales. 4 (106), 93-106.

Cuvardic García, D. y Vargas Castro, E. (2010). Recursos lingüísticos en la titulación periodística costarricense: el caso de La Nación y el diario La Extra. Revista de Filología y Lingüística. 36 (1), 207-232.

De Benito, E. (3 de julio de 2005). Una multitudinaria marcha celebra en Madrid la igualdad de derechos de los homosexuales. El País. Recuperado de http://elpais.com/ diario/2005/07/03/sociedad/1120341601_850215.html

Douglas, S., Pecora, N. y Guback, T. (1985). Work, workers and the workplace: is local newspaper coverage adequate? Journalism Quarterly. 62 (4), 855-60.

Entman, R. (1993). Framing: Towards a Clarification of a Fractured Paradigm. Journal of Communication. 43 (4). 51-58.

Entman, R. M. y Rojecki, A. (1993). Freezing out the public: Elite and media framing of the U.S. antinuclear movement. Political Communication. 10 (2), 151-167.

Ericson, R. V., Baranek, P. M. y Chan, J. B. L. (1989). Negotiating control: A study of news sources. Toronto, Ontario, Canada: University of Toronto Press.

Ericson, R. V., Baranek, P. M. y Chan, J. B. L. (1991). Representing Order: Crime, Law, and Justice in the News Media. Milton Keynes, England: Open University Press.

Fallas, H. (26 de febrero 2007). Frente promete marcha pacífica hoy. La Nación. Recuperado de http://www.nacion.com/nacional/Frente-promete-marcha-pacifica-hoy_0_887711305.html

Fallas, H. (26 de febrero de 2007). Marcha tuvo poca afectación en servicios públicos. La Nación. 26/2. Recuperado de http://www.nacion.com/archivo/Marcha-poca-afectacionservicios-publicos_0_887711289.html 
Fallas, H. (22 de julio de 2007). Grupos del 'no' planean más manifestaciones. La Nación. Recuperado de http://www.nacion.com/nacional/politica/Grupos-planeanmanifestaciones_0_916908410.html

Gamson, W. A. y Modigliani, A. (1989). Media discourse and public opinion on nuclear power: a constructionist approach. American Journal of Sociology. 95 (1), 1-37.

Gitlin, T. (1980). The Whole World is Watching: Mass Media in the Making and Unmaking of the New Left. Berkeley: University of California Press.

Golcher, R. (14 de agosto de 2004). La APSE luchará contra TLC. La Nación. Recuperado de http://wvw.nacion.com/ln_ee/2004/agosto/14/pais5.html

Hansen, A., Cottle, S., Negrine, R. y Newbold, C. (1998). Mass Communication Research Methods. London: Macmillan.

Hartmann, P. (1979). News and public perceptions of industrial relations. Media, Culture and Society. 1 (3), 255-70.

Herrera, M. (2004). Partidarios del TLC triplican a opositores. La Nación. Recuperado de http://wvw.nacion.com/ln_ee/2004/marzo/17/pais1.html

Knight, G. (1982). News and ideology. Canadian Journal of Communication. 8 (4), 15-41.

Kress, G. y Van Leewen, T. (2006 [1996]). Reading Images. The Grammar of Visual Design. London: Routledge.

Lafuente Pérez, P. y Zugasti Azagra, R. (2012). La irrupción del 15-M: la cobertura en El País y en El Mundo. Periodismo político en España: concepciones, tensiones y elecciones. (pp. 120-132). La Laguna: Sociedad Latina de Comunicación Social.

Leal, D. (1 de junio de 2004). Trabajadores marcharon contra el TCL. La Nación. Recuperado de http://wvw.nacion.com/ln_ee/2004/junio/01/pais2.html

Leal, D. (2 de mayo de 2007). Trabajadores marcharon por TLC y referendo. La Nación. Recuperado de http://www.nacion.com/nacional/Trabajadores-marcharon-TLCreferendo_0_900709998.html

Loaiza, V. (13 de octubre de 2003). Gremios marcharán contra TLC. La Nación. Recuperado de http://wvw.nacion.com/ln_ee/2003/octubre/13/pais4.html

López del Ramo, J. y Torregrosa, J. F. (2011). Documentalismo fotográfico y conflictividad sociolaboral. Análisis del caso de la huelga general de 2010 en los diarios españoles. Documentación de las Ciencias de la Información. 34, 277-290.

Lozano, J. C. (1994). Hacia la reconsideración del análisis de contenido en la investigación de los mensajes comunicacionales. En C. Cervantes Barba y E. Sánchez Ruiz (Coords.). Investigar la Comunicación: Propuestas Iberoamericanas. (pp. 135-158). Guadalajara: Universidad de Guadalajara. Centro de Estudios de la Comunicación y de la Información.

Marshall, T. (1977). Trouble at t'Millpond -Farmworkers in the media. En P. Beharrel y G. Philo (Eds.). (pp. 50-59). Trade and the media. London: MacMillan Press.

Martínez Nicolás, M. (1994). La construcción de la crisis del SIDA en la información periodística. Análisis del discurso informativo sobre el SIDA en Cambio 16, Tiempo de Hoy e Interviú (1982-1992). Bellaterra, Barcelona: Universidad Autónoma de Barcelona. 
McLeod, D. M. y Detenber, B. H. (1999). Framing effects of television news coverage of social protest. Journal of Communication. 49 (3), 3-23.

Meyer, D. S. y Staggenborg, S. (1996). Movements, countermovements, and the structure of political opportunity. American Journal of Sociology. 101 (6), 1628-60.

Mora, A. L. (20 de noviembre de 2007). Sindicatos anuncian paros y marchas. La Nación.

Morales Morante, F. (2014). Análisis del tratamiento informativo en noticias de televisión. Estudio de caso de la huelga de profesores en España y Perú. Correspondencias y Análisis. 4, 191-214.

Murillo, A. y Agüero, M. (1 de octubre de 2007). El NO evoca a próceres para pedir 'fusilar' a TLC en las urnas. La Nación. Recuperado de http://wvw.nacion.com/ln_ee/2007/ octubre/01/pais1259895.html

Oviedo, E. (24 de octubre de 2006). Pacífica marcha contra el TLC. La Nación. Recuperado de http://www.nacion.com/nacional/Pacifica-marcha-TLC_0_862713797.html

Oviedo, E. (26 de febrero de 2007). Marcha contra el TLC termina sin incidentes. La Nación. Recuperado de https:/www.nacion.com/archivo/marcha-contra-el-tlc-termina-sinincidentes/APJFPVSBGZFORJUFJDLOKEPEFE/story/

Oviedo, E. (26 de febrero de 2007). Miles de estudiantes sin clases por marcha, pero apoyo de docentes a movimiento es parcial. La Nación. Recuperado de http://wvw.nacion.com/ ln_ee/2007/febrero/26/ultima-sr1009878.html

Oviedo, E. (27 de febrero de 2007). Marcha pacífica reunió a 23.500 opositores al TLC. La Nación. Recuperado de http:/www.nacion.com/nacional/Marcha-pacifica-reunioopositores-TLC_0_887911295.html

Piquer Vidal, A. (2013) Puntos de vista sobre el 15M. Aspectos pragmaestilísticos y deriva ideológica. Estudios sobre el Mensaje Periodístico. 19 (1), 223-234.

Rada, S. E. (1977). Manipulating the media: a case study of a Chicano strike in Texas. Journalism Quarterly. 54 (1), 109-113.

Romero Lizama, P. (2013). Análisis crítico de la representación informativa, de Camila Vallejo y el Movimiento Estudiantil chileno 2011 en el diario Las Últimas Noticias. Estudios sobre el Mensaje Periodístico. 19 (2), 871-888.

Shoemaker, P. J., Chang, T. y Brendlinger, N. (1987). Deviance as a Predictor of Newsworththiness: Coverage of International Events in the U.S.Media. Communication Yearbook. 10, 348-365.

Snow, D. A. y Benford, R. D. (1988). Master Frames and Cycles of Protest. En A. D. Morris y C. McClurg Mueller (Eds). Frontiers in Social Movement Theory. (pp. 133-155). New Haven and London: Yale University Press.

Stevenson, R. L. y Greene, M. T. (1980). A reconsideration of bias in the news. Journalism Quarterly. 57 (1), 115-21.

Swart, W. J. (1995). The League of Nations and the Irish Question: Master frames, cycles of protest, and "master frame alignment". The Sociological Quarterly. 36 (3), 465-481.

Tarrow, S. (1988). Mentalities, Political Cultures, and Collective Action Frames. Constructing Meaning through Action. En A. D. Morris y C. McClurg Mueller (Eds). Frontiers in Social Movement Theory. (pp. 174-202). New Haven and London: Yale University Press. 
Terkildsen, N. y Schnell, F. (1997). How Media Frames Move Public Opinion: An Analysis of the Women's Movement. Political Research Quarterly. 50 (4), 879-900.

Tuchman, G. (1982). La producción de la noticia. Un estudio sobre la construcción de la realidad. Barcelona: Editorial Gustavo Gili.

Tuchman, G. (1983). La producción de la noticia. Estudio sobre la construcción de la realidad. Barcelona: Editorial Gustavo-Gili.

Umaña, J. (23 de febrero de 2007). Arias confía en una marcha sin violencia. La Nación. Recuperado de http://www.nacion.com/nacional/Arias-confia-marchaviolencia_0_887111358.html

Valera Ordaz, L. (2016). El sesgo mediocéntrico del framing en España: una revisión crítica de la aplicación de la teoría del encuadre en los estudios de comunicación. Zer-Revista de Estudios de Comunicación. 21 (41), 13-31.

Van Dijk, T. (2003). Racismo y discurso de las élites. Barcelona: Editorial Gedisa.

Van Zoonen, E. (1992). The women's movement and the media: constructing a public identity. European Journal of Communication. 7 (4), 453-76.

Velásquez, C. A. (2004). Cubrimiento televisivo-noticioso de protestas sociales en Colombia. Palabra Clave. 10, 9-37.

Villalobos, C. (20 de octubre de 2003). Sindicatos en pulso hoy contra TLC. La Nación. Recuperado de http://wvw.nacion.com/ln_ee/2003/octubre/20/pais4.html

Villalobos, C. (13 de marzo de 2007). Indisposición general para participar en bloqueos. La Nación. Recuperado de http://www.nacion.com/nacional/Indisposicion-generalparticipar-bloqueos_0_890710935.html

Villalobos, C. (23 de agosto de 2007). Hoy, primer debate en TV entre el Sí y el NO. La Nación. Recuperado de http://www.nacion.com/nacional/politica/Hoy-primer-debateTV_0_923307736.html_

Villegas J. y Ávalos, Á. (27 de agosto de 2004). Débil apoyo de empleados públicos a paro nacional. La Nación. Recuperado de http://wvw.nacion.com/ln_ee/2004/agosto/27/ portada.pdf

Villegas, J. y Herrera, B. (21 de octubre de 2003). Marcha llenó 200 metros del Paseo Colón. La Nación. Recuperado de http://wvw.nacion.com/ln_ee/2003/octubre/21/pais5.html

Villegas, J. (18 de noviembre de 2005). Marcha pacífica contra TLC. La Nación. Recuperado de wvw.nacion.com/ln_ee/2005/noviembre/18/pais0.html

Walsh, G. (1988). Trade unions and the media. International Labour Review. 127 (2), 205-220.

Wolfsfeld, G. (1991). Media, Protest, and Political Violence. A Transactional Analysis. Journalism and Communication Monographs. 127. 